\section{$\underset{\substack{\text { hommes } \\ \text { \& migrations }}}{ }$}

\section{Hommes \& migrations}

Revue française de référence sur les dynamiques

migratoires

\section{$1308 \mid 2014$}

Les Paris des migrants

\title{
Échappé aux griffes de la tribu
}

\section{Mustapha Harzoune}

\section{(2) OpenEdition \\ Journals}

\section{Édition électronique}

URL : http://journals.openedition.org/hommesmigrations/3024

DOI : 10.4000/hommesmigrations.3024

ISSN : 2262-3353

\section{Éditeur}

Musée national de l'histoire de l'immigration

\section{Édition imprimée}

Date de publication : 1 octobre 2014

Pagination : 180-183

ISBN : 978-2-919040-29-2

ISSN : 1142-852X

\section{Référence électronique}

Mustapha Harzoune, «Échappé aux griffes de la tribu », Hommes \& migrations [En ligne], 1308 | 2014, mis en ligne le 26 juin 2015, consulté le 24 septembre 2020. URL : http://journals.openedition.org/ hommesmigrations/3024; DOI : https://doi.org/10.4000/hommesmigrations.3024 


\title{
ÉCHAPPÉ AUX GRIFFES DE LA TRIBU
}

\author{
MUSTAPHA HARZOUNE
}

I es manifestations de l'été, les commentaires Lqui les ont accompagnées finiraient de déstabiliser ceux pour qui l'idéologie dénonçant l'islamophobie serait propos de paranos et d'excités en mal de reconnaissance. Dans le viseur non pas l'intervention israélienne à Gaza mais ses retombées sur le vivre-ensemble hexagonal. L'antienne est rabâchée : l'intégration à la papa butterait aujourd'hui sur la frilosité des nouveaux immigrés - autre culture, autre religion - à plonger dans la marmite nationale. Face à ces réfractaires, il faudrait s'inquiéter, réagir, fustiger, expulser et tout le toutim. On en oublierait que Marianne daubait aussi les Polacks, Ritals, Espingouins et autres.

Deux communautarismes se seraient défiés cet été : communautarisme "arabe" versus communautarisme juif. De ce dernier, il est rarement question. On s'abstient. On se retient. On esquive. Pourtant, il est tout aussi réel - et tout aussi fantasmé - que le premier. Le hic, c'est que, comme sur d'autres questions ou tensions qui épuisent la société et minent le vivre-ensemble, il est plus commode de céder à la facilité, à l'impulsif, au machinal, et de détremper le raisonnement de force émotions lacrymales. Le 8 juillet 2014, Israël bombardait Gaza, la campagne "Bordure protectrice” commençait. Elle allait durer jusqu'à la signature, le 26 août, d'un accord de cessez-lefeu. L'armée israélienne laissera derrière elle plus de 2200 victimes, majoritairement civiles, et plusieurs milliards de ruines. Comme le bras armé a toujours et partout besoin de justifier l'usage de la force, Goliath entendait - comme en 20082009 déjà - faire cesser les tirs de roquettes lancées depuis Gaza. Ce déchaînement de feu eut des répercussions jusqu'en France. Des milliers de citoyens se mobilisèrent, pour défendre qui Tsahal, qui la cause palestinienne. La majorité pour simplement rappeler les principes constitutifs d'un vivre-ensemble planétaire, éternels principes au fondement du respect de toute humanité. Ces derniers furent-ils seulement entendus, dès lors que la doxa les étiqueta de "manifestants propalestiniens" ou pire "pro-Hamas". La messe était dite et l'agora abandonnée aux belliqueux et aux idiots, plus ou moins utiles.

\section{Lignes de fracture}

Ces manifestants (et contre-manifestants) n'auraient laissé dans leur sillage qu'un fond de haine, des débordements, des slogans et propos racistes. Ainsi de la manifestation du 13 juillet, n'a-t-on retenu que les "attaques contre deux synagogues". Dans Libération du 15 juillet, Haïm Korsia, grand rabbin de France, déclare : "Au moment de la dissolution de la manif propalestinienne autour de la Bastille, des groupes de manifestants ont voulu converger vers la synagogue de la rue des Tournelles, et les forces de police les en ont empêchés. Ils sont allés ensuite à la synagogue de la rue de la Roquette pour l'attaquer avec une grande violence." Haïm Korsia parle de "traumatisme", "d'une foule complètement hystérique et dangereuse" et, citant un homme âgé de 90 ans, il évoque "la Nuit de cristal". Avant de renvoyer les manifestants à ce que Deleuze qualifiait de "fiction" (voir le blog de Yvan Najiels hébergé par Médiapart le 3 août) : "Il ne faudrait pas être dupes du prétexte géopolitique : l'antisionisme a les mêmes habits que l'antisémitisme." À cela le grand rabbin ajoute, artiste 
de la rhétorique et de la dialectique : "ll y a eu des slogans insupportables, inqualifiables, il faut oser le dire. Je crois qu'il faut dire les choses sinon c'est l'ensemble du système qui constitue la société française qui s'écroule. C'est une haine de la France, une haine du vivre-ensemble."

La glissade maîtrisée amalgame les manifestants à des "propalestiniens", de là à des "antisionistes" et donc à des "antisémites", doublement dangereux pour leur "haine" du "vivre-ensemble" et de la France. Rien moins. Dans un entretien remis à l'ordre du jour sur les réseaux sociaux, I'historien Shlomo Sand, qui condamnait un autre "pilonnage de Gaza", tenait un raisonnement différent : "Ce qui me choque plus que jamais, c'est que cet État que j'ai servi comme soldat durant deux guerres, et qui se définit depuis sa Déclaration d'indépendance en 1948 comme l'État de tous les juifs, appartienne davantage à Bernard-Henri Lévy qu'à mes amis universitaires qui vivent ici, payent leurs impôts ici, mais sont d'origine arabe. Qu'est-ce que ça veut dire être sioniste quand on vit en France, qu'on ne veut pas vivre sous l'autorité juive, et qu'on s'identifie au pire de la politique des dirigeants d'Israël ? Ça veut dire contribuer à la montée de l'antisémitisme" (Télérama, n 3081, 31 janvier 2009).

Les condamnations unanimes - et justifiées des débordements et des violences d'une minorité ont contribué à rendre invisibles et inaudibles des milliers de manifestants. Ils ont travesti non pas la réalité, mais évacué des radars médiaticopolitiques celles et ceux qui défilaient justement au nom du vivre-ensemble. Et quand des manifestations furent interdites, les libertés publiques et les règles démocratiques frémirent. Pourtant, ces mobilisations n'étaient-elles pas, dans leur composition et leur expression, bien plus républicaines que ce qu'il a été dit et écrit ? Le 14 juillet, à propos de la même manifestation, Michèle Sibony, de I'Union juive française pour la paix (UJFP), témoigne dans un texte intitulé "Intox, mensonge et presse écrite" adressé au "rédacteur en chef adjoint de Rue 89": "J'ai assisté à de nombreuses scènes émouvantes: une de nos adhérentes, jolie dame rousse qui a connu la déportation et qui milite entre autres très activement pour les droits des étrangers en France, a attiré, à cause du badge UJFP qu'elle portait, de nombreuses personnes qui voulaient la prendre en photo, l'embrasser. 'Madame, vous ne pouvez pas savoir le bien que vous nous faites, c'est si important que vous soyez là. Vous êtes pour nous un symbole, celui de la paix. Cette histoire n'a rien de religieux, il ne s'agit pas de juifs et de musulmans...' Pas un mot vu, lu ou entendu contre les juifs, pas une affiche, pas un slogan, rien..."

\section{Les médias dans le viseur}

Revenant sur les affrontements devant la synagogue de la Roquette, Michèle Sibony apostrophe le journaliste de Rue 89 parce qu'il cite "sans sourciller" un "témoignage de la LDJ", la Ligue de défense juive, qui appelait à un rassemblement "en soutien à Israël devant la synagogue de la Roquette, sur leur site, avec ce titre éloquent : 'Keep calm and kill Hamas (...)'. Alors je vais vous dire ce quej'ai vu, moi, pauvre juive infidèle, écrit-elle: Sur le boulevard Beaumarchais, à peu près à la hauteur de Chemin vert, 4 ou 5 types de la Ligue de défense juive montés sur un banc, complètement entourés et protégés par deux rangs serrés de CRS, qui jetaient projectiles et insultes sur la foule, et les services d'ordre, et les responsables calmant les manifestants, 'Ne vous énervez pas, ne répondez pas aux provocations, c'est ce qu'ils attendent..., et bien sûr, lors de la dispersion, il y a eu des courses et des bagarres à l'entrée de la rue de la Roquette... comme prévu si j'ose dire. Et surtout, j'ai aussi entendu la foule des manifestants crier depuis Barbès jusqu'à la Bastille. 'Médias français, montrez la vérité,' 'Le peuple français veut la vérité.' Et j'étais fière aujourd'hui de ce peuple-là, de mon peuple."

Dans Le Monde du 4 août, Rony Brauman, Régis Debray, Edgar Morin et Christiane Hessel (veuve de Stéphane Hessel) s'adressaient à François Hollande, l'enjoignant de "résister au mensonge": "Les appels pieux ne suffisent pas plus que les renvois dos à dos qui masquent la terrible disproportion de forces entre colonisateurs et colonisés depuis qua- 


\section{KIOSQUE}

rante-sept ans. L'écrivain et dissident russe Alexandre Soljenitsyne (1918-2008) demandait aux dirigeants soviétiques une seule chose: 'Ne mentez pas.' Quand on ne peut résister à la force, on doit au moins résister au mensonge. Ne vous et ne nous mentez pas, monsieur le Président. (...) Ce ne sont certes pas des Francais qui sont directement en cause ici, c'est une certaine idée de la France dont vous êtes comptable, aux yeux de vos compatriotes comme du reste du monde." Pour le journaliste et essayiste Akram Belkaïd, "dès lors qu'il s'agit d'Israël, les médias français, notamment les chaînes télévisées d'information continue, sont terrorisées à l'idée de commettre un impair qui ouvrirait la porte à des accusations d'antisémitisme" (EI Watan, 4 août). Le 13 juillet, dans Libération, Daniel Schneidermann fournissait un éclairage sur ces "précautions", cette "objectivité", cette "neutralité" journalistiques qui gomment toutes perspectives historiques et résument les faits à "ce sont les Palestiniens qui ont commencé, et les Israéliens ne mènent que des 'représailles"'. II écrit : "En tenir un. Un seul, une fois. Le regarder dans les yeux. Lui expliquer gentiment. Surtout, gentiment. Lui expliquer qu'on n'est pas spécialement propalestinien. Pas davantage que proisraélien. Qu'à la limite, on s'en fiche. Qu'on ne veut même plus savoir qui a tort ou raison, qui a commencé, qui était là le premier, on s'est assez tiré les neurones dans tous les sens, on est fatigué d'essayer de départager. Mais tout de même, qu'on aimerait simplement comprendre. (...) Écouter toutes ses réponses (...). Regarder toutes ses justifications s'autodissoudre les unes après les autres, pour laisser à nu la seule réponse possible: c'est parce qu'on a toujours fait comme ça, et on fait comme ça parce que tous nos confrères sur place vivent à Jérusalem ou à Tel-Aviv, en tout cas pas en Palestine, et que nous sommes donc culturellement, économiquement, affectivement, intégrés à la société israélienne, et pas à la société palestinienne, même si c'est largement à notre insu, comme d'habitude."

\section{Dérapage (non) contrôlé}

Jeune. Immigré. Arabe. Français musulman. Musulman. Propalestinien. Intégriste. Haine du vivre-ensemble. Ennemi de la France. Le diagnostic du refus d'intégration cher à Éric Zemmour ou à Natacha Polony court le Landerneau. Dans une "lettre à un jeune compatriote musulman" (Le Figaro, 28 juillet), cette dernière décide de s'adresser - en le tutoyant ! - à ce destinataire par elle fabriqué et qu'elle veut, d'entrée, rassurer : "Je n'ai aucun 'problème' avec l'islam. Bien au contraire, ce mot éveille en moi le souvenir de l'immense civilisation arabo-musulmane qui fut au XIII siècle le phare du monde" (sic). Mme Polony est chagrinée par "ceux qui s'en réclament pour imposer leur intolérance et poursuivre un objectif politique, celui notamment de modifier l'équilibre des droits et des devoirs dans ce vieux pays qui avait à peu près réglé la question des religions". II faut humer toutes les saveurs de cet "à peu près" après les mobilisations contre le mariage pour tous, l'homophobie œecuménique, l'avortement qui continue de donner des boutons... La ci-devant prof renvoie "son" jeune et toujours imaginaire compatriote musulman à "trois jeunes étudiants" qui lui déclaraient "qu'ils n'iront pas lire les mythes de la Genèse parce que 'leur religion leur interdit d'ouvrir ce livre"' ou même à Tariq Ramadan qui, évacuant toute "culture musulmane", distribuerait le monde en "musulmans" et "non-musulmans". Et Natacha Polony de conclure : "Quand un fou ou un frustré endoctriné tue en se réclamant de l'islam, j'espère entendre ta voix, cher compatriote, me dire que tu récuses cette façon abjecte d'enrôler ta religion. (...) Vivre en bonne entente, cher compatriote, c'est avoir confiance en l'autre, qui est un semblable. Et nous avons un destin commun dans ce pays, la France, qui a développé un compromis social et politique que nous devons préserver toi et moi." 
"Trois jeunes Françaises - une journaliste et deux doctorantes"(Nadia Henni-Moulai, Hanane Karimi, Fatima Khemilat) - ont, dans une tribune sur le site de L'Obs le 2 août, renvoyé fissa Natacha Polony à "ses fantasmes". Notamment ce "fantasme coutumier, celui d'une communauté musulmane homogène et réifiée, piétinant comme un seul homme 'léquilibre des droits et des devoirs de ce vieux pays'. (...) Mais 'le musulman' auquel Natacha Polony se confie présente quelques caractéristiques. (...) Les apartés faits par Natacha Polony sur les chrétiens persécutés d'Irak, sur Tariq Ramadan suisso-égyptien ou les 'djihadistes' syriens montrent cette tendance à l'imposition d'une solidarité négative et d'une responsabilité collective des Arabomusulmans'. L'essayiste l'enjoint, tout en rappelant la francité de son interlocuteur imaginaire, à se positionner quant aux 'dérives' du 'monde arabe'." Le même jour, dans Le Nouvel Obs, une tribune signée d'un pseudo, Lilith, qui se présente comme "Française d'adoption, Arabe d'origine et ex-musulmane", s'oppose avec virulence à la réponse des trois auteures: "La seule ignorance que je vois, mesdames, c'est la vôtre. Cette ignorance s'appelle 'le déni.' (...) Ce que vous refusez de comprendre, c'est que non seulement la France, mais également le monde entier, fait face aujourd'hui à divers courants idéologiques et au caractère politique, tous issus d'une seule source quasiment, la doctrine islamiste rigoriste d'Ahmad Ibn-Hanbal. (...) Nous ne sommes plus (...) dans le clivage 'Blanc-chrétien-athée'/'basané-musulman'. Nous sommes au coeur d'un conflit idéologique et politique, sous couvert de religion. C'est donc à juste titre que Mme Polony interpelle ce jeune citoyen musulman. Et je l'interpelle de même!"

\section{Solidarité républicaine}

Tandis qu'en France un manifestant qui défile pour dire non au massacre et à la violence est un "propalestinien", que des jeunes, renvoyés à une appartenance réelle ou fantasmée, doivent subir un procès en suspicion faute de se désolidariser des fous de Dieu qui se repaissent d'abord de victimes musulmanes, intelligence et sensibilité fleurissent. Ailleurs. Sur des terres dédaignées. Ainsi Kamel Daoud ose, le 13 juin, en Algérie, écrire "pourquoi je ne suis pas 'solidaire' de la Palestine". "Non à la 'solidarité' sélective. Celle qui s'émeut du drame palestinien parce que ce sont des Israéliens qui bombardent. Et qui, donc, réagit à cause de l'ethnie, de la race, de la religion et pas à cause de la douleur. (...) Non donc à la 'solidarité' par conditionnement religieux et 'nationaliste'. (...) Le drame palestinien a été 'arabisé' et islamisé à outrance, au point où maintenant le reste de l'humanité peut se sentir débarrassé du poids de cette peine. (...) Solidarité n'est pas choix, mais élan total envers toutes et tous. Solidarité avec l'homme, partout, contre I'homme qui veut le tuer, le voler ou le spolier, partout. Solidarité avec la victime contre le bourreau parce qu'il est bourreau, pas parce qu'il est israélien, chinois ou américain ou catholique ou musulman. (...)." Le chroniqueur est pour l'autre solidarité : "celle totale et entière et indivise. Celle qui fait assumer, par votre dignité, au reste du monde, sa responsabilité envers une question de colonisation, pas de croyances. (...). Ce que fait Israël contre Gaza est un crime abject. Mais nos 'solidarités' sont un autre qui tue le Palestinien dans le dos." Kamel Daoud fut traité de "collabo, harki, sioniste, sale juif, kafir, mécréant, apostat, vendu, youpin! traitre, salaud, r'khis, lâche, sale rabbin, merdeux, valet de BHL, suppôt d'Israël...", rappelle Mohamed Kacimi (choufchouf.com, 26 juillet). Histoire de titiller quelques fantasmes qui prospèrent en Occident, jusque dans des têtes bien faites et de s'émanciper des communautarismes, il écrit :"Kamel ne parle pas au nom des autres des Algériens, et encore moins des Arabes et des musulmans, il est enfin un individu, un intellectuel, un romancier échappé aux griffes de la tribu qui s'en fiche des mots de la tribu parce qu'il a les siens et qu'il peut dire merde à la tribu (...)."Toutes les tribus. 